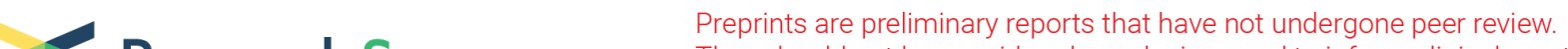

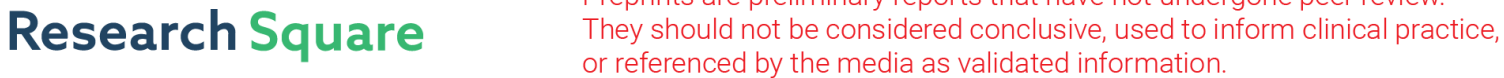

\section{Effects of Balance and Timing Constraints on Performance During a Pointing Task with Target Uncertainty}

\section{Christopher Moya-Jofré}

Motor Learning and Neurorehabilitation Lab, Faculty of Medicine, Universidad de Chile Juan José Mariman

Department of Physical Therapy, Faculty of Arts and Physical Education, Universidad Metropolitana de Ciencias de la Educación.

\section{Julio Torres-Elgueta}

Department of Physical Therapy, Faculty of Medicine, Universidad de Chile.

\section{Trinidad Bruna-Melo}

Motor Learning and Neurorehabilitation Lab, Faculty of Medicine, Universidad de Chile

\section{Fernanda Aleitte-Leyton}

Motor Learning and Neurorehabilitation Lab, Faculty of Medicine, Universidad de Chile

\section{José Carrasco-Plaza}

SCIAN-Lab, Faculty of Medicine, Universidad de Chile

\section{Cristian Muñoz-Puelman}

Motor Learning and Neurorehabilitation Lab, Faculty of Medicine, Universidad de Chile

\section{Esteban Aedo-Muñoz}

Centro de Alto Rendimiento, Av. Pedro de Valdivia, Ñuñoa.

\section{Pablo Ignacio Burgos ( $\nabla$ pburgos@uchile.cl)}

Department of Neuroscience, Faculty of Medicine, Universidad de Chile.

\section{Research Article}

Keywords: balance, reaching, online correction, feedback time

Posted Date: February 7th, 2022

DOI: https://doi.org/10.21203/rs.3.rs-1292717/v1

License: (c) (1) This work is licensed under a Creative Commons Attribution 4.0 International License. Read Full License 


\section{Abstract}

Introduction: In dynamic tasks, we must make online corrections when the position of the body or objects change, which alters the feedforward and feedback mechanisms. This research aimed to determine the effect of postural stability and correction times on a pointing task with uncertain targets.

Methodology: 19 young subjects performed a pointing task toward targets that exhibited a change in position (at 200,400, or 600 ms after) while remaining in a bipedal-firm, a bipedal-foam, and a unipedalfoam surface. Kinematics parameters were recorded during pointing.

Results: What affects the precision and duration of pointing is the target change timing, not the postural condition. The target change timing and the postural condition affect the movement strategy, expressed in kinematic parameters of the center of pressure (CoP), the upper-trunk, and the index finger. However, the CoP control responded to the postural demands, while the finger and trunk control responded to the postural requests and the target change timing.

Conclusion: Different postural strategies allow the achievement of a pointing task despite the changes in postural conditions. Moreover, the later the target changes, the more challenging it is to correct the reaching trajectory, achieved by a prioritization of the endpoint control.

\section{Introduction}

The bipedal posture has allowed humans to interact with the external environment by manipulating objects while maintaining balance. In our daily activities, it is common to carry out these manipulation and reaching tasks in dynamic situations (movements of objects or the body) without significant difficulties ${ }^{1}$. Different mechanisms are involved in maintaining a stable sensorimotor context for the successful execution of tasks, particularly in these dynamic contexts ${ }^{2}$. Postural control mechanisms, which work in parallel with those for the control of eye-hand coordination, maintain postural stability during manual tasks, avoiding a loss of manual precision due to postural disturbances ${ }^{3-7}$. Postural adaptations become so effective that even postural oscillation may be less during higher-demand tasks than during low-demand tasks ${ }^{8}$.

The reaching task consists of a succession of steps to control the motion, which involve the target location, the generation of a motor plan, and finally, the execution 9,10 . Sensory information and internal models are used to plan the movement (feedforward mechanism) and correct it (feedback mechanism) 11-13. The difference between the predictive and current sensory inputs (sensory error) triggers an online modification in the motor command ${ }^{8}$. Previous works have shown the influence of feedback and feedforward mechanisms in reaching tasks ${ }^{12,14}$. However, what remains unclear is how these mechanisms operate in situations with uncertain contexts (for example, when the location of the target changes during the execution of the reaching task) ${ }^{13}$. 
Among the various options for studying reaching tasks in a dynamic situation, the double-step paradigm has been widely used ${ }^{15-17}$. In this paradigm, the target is displaced while the hand is executing the reaching; therefore, the participants must update the movement in progress. One of the advantages of this paradigm is the control of the target change time, which allows exploring the contribution of the different control mechanisms (particularly late changes decrease the time available for feedback adjustments) ${ }^{18}$. Previous work has shown that reaching updates are often completed in the range of 100 to $150 \mathrm{~ms}^{15,17-20}$.

Most of the literature related to reaching tasks with online correction has been derived from experiments performed with subjects in a sitting position ${ }^{19}$. Reaching tasks with correction in bipedal positions have also been used ${ }^{21,22}$. However, the effect of postural adjustments themselves on the reaching mechanisms in more challenging postural situations such as smaller or unstable bases of support has not been studied ${ }^{21}$.

These experiments suggest high flexibility to adapt the reaching task to different postural conditions or correction times ${ }^{15,17-20}$. Nevertheless, it is not known if different degrees of postural stability determine the effectiveness of the feedforward and feedback mechanisms during reaching with different correction times. Based on previous studies of dual-task paradigms, these results suggest that balance or reaching performance should be affected in dynamic reaching situations with postural instability ${ }^{23,24}$.

This research aimed to determine the effect of different postural stability conditions on performance during a pointing task toward uncertain targets with different change times. We hypothesized that conditions with less postural stability affect pointing performance, particularly toward targets with late changes in their location.

To test the hypothesis, 19 young subjects performed a pointing task that exhibited a change in position (at 200,400, or $600 \mathrm{~ms}$ after the initiation of pointing), while staying in a bipedal-firm, a bipedal-foam, and a unipedal-foam surface. The position of the target was modified in each trial, with a random order in three different times $(200,400,600 \mathrm{~ms})$. Variables related to performance were analyzed with respect to the target (time and error) and the kinematic parameters of the pointing (ranges of displacement and velocity of the center of pressure, index finger, and upper trunk).

\section{Results}

\section{The postural condition affects stability}

To evaluate which postural conditions produce greater demands for stability (bipedal-firm, bipedal-foam, or unipedal-foam), we evaluated the anteroposterior (A-P) and mediolateral (M-L) displacement of the CoP before the onset of the pointing $(-500 \mathrm{~ms}$ to $0 \mathrm{~ms})$. The results of the A-P range of the CoP displacement (Figure 2A) did not show differences between the postures $(F(17 / 34)=1.78, p=0.18)$. The $M-L$ range (Figure $2 B$ ) showed differences between the postures $(F(17 / 34)=22.07, p \leq 0.001)$. The 
unipedal-foam posture was more unstable than the bipedal-firm $(p \leq 0.001)$ or bipedal-foam $(p \leq 0.001)$ postures.

To verify that the near-surface instability represented by CoP was not dissipated by postural adjustments of the lower extremities and trunk, we evaluated the displacement of the upper trunk (C7 spinous process marker). Similar results to the CoP were found without differences between the postures in A-P (F (17/34) $=3.12, p=0.07)$ and with differences in the $M-L$ range (Figure $2 C-D)(F(17 / 34)=51.60, p \leq 0.001)$. The unipedal-foam posture was significantly more unstable than the bipedal-firm $(p \leq 0.001)$ or bipedal-foam postures $(p \leq 0.001)$.

\section{Pointing performance is affected by the target change timing and not the postural condition}

To evaluate the pointing performance on the screen, we measured the motion time and the error for the target. The movement times were around $1.2 \mathrm{~s}(1 \mathrm{~s}$ to $1.8 \mathrm{~s})$, and the error was about $0.4 \mathrm{~cm}(0.3 \mathrm{~cm}$ to $1 \mathrm{~cm})$. We did not perform statistics on the original data but on the normalized data with respect to 15 baseline trials where the target always remained static on the screen. We also used a metric that combines error and time to determine the composite effect. The statistical details of each comparison are presented in Table 1.

Motion time was shorter during the task with target change than during the baseline condition of no change (negative z-score values in Figure $3 \mathrm{~A}$ ). Motion time increased as change time increased. When comparing the effect of the target change time on the finger movement time, significant differences were observed between the $600 \mathrm{~ms}$ of change and the other change times $(F(2.34)=26.01, p \leq 0.001)$. Differences were also observed to a lesser extent between the other change times $(0,200$ and $400 \mathrm{~ms})$. This demonstrates that motion time increases significantly in the face of late target changes.

When comparing the effect of postural stability on motion time, no significant differences were observed $(\mathrm{F}(2.36)=0.085, \mathrm{p}=0.82)$.

The pointing error was greater compared to the baseline condition (positive $z$ score values in Figure 3B), with an increase in the error as the target change time increased. Significant differences were found between the effect of the target change times and the pointing error $(F(3.54)=12.33, p \leq 0.001)$ for 600 $\mathrm{ms}$. With a shorter correction time, the pointing error increased. When comparing the effect of postural condition on pointing error, no significant differences were observed $(F(6.108)=0.17, p=0.84)$.

When comparing the values of the composite metric of error and movement time (Figure $3 \mathrm{C}$ ), we could observe that the target change at $600 \mathrm{~ms}$ differed from the other change times $(0,200$ and $400 \mathrm{~ms})$, ( $F$ $(3.54)=19.47, \mathrm{p} \leq 0.001)$. It could also be observed that the target change times of $200 \mathrm{~ms}$ and $400 \mathrm{~ms}$ did not differ from the no change $(0 \mathrm{~ms})$ during the task with online correction. When comparing the effect of postural stability on the composite metric, no significant differences were observed $(F(2.36)=$ $0.005, p=0.99$ ).

\section{Both the target change timing and the postural condition affect the movement strategy}


The pointing movement strategy was evaluated through displacement ranges and mean speed of the index finger, upper thorax (marker at C7), and CoP during movement of the index finger from the xiphoid process to the screen (Figure 4). Based on section 2, we only compared the change time of $600 \mathrm{~ms}$ vs. 0 $\mathrm{ms}$, which presented the main differences.

The index finger movement strategy was not affected in the A-P displacement by the target time ( $F(1.17)$ $=0.54, p=0.47)$ or by the postural condition $(F(2.34)=2.60, p=0.10)$, which was the main requirement of the task (Figure $4 \mathrm{~A}$ ). The M-L displacement showed that there was a modification of the strategy in relation to the change time $(F(1,17)=93.38, p \leq 0.001)$ and to the postural condition $(F(2.34)=7.94, p=$ $<0.001)$. M-L displacement increased while the change time and postural instability increased (Figure $4 \mathrm{~B})$. There was a decrease in the mean speed of the finger in the trials with online correction in the bipedal-firm condition ( $F(1.17) 4.44, p=0.06)$ (Figure 4C).

For the control of the upper trunk, a strategy of significant decrease in the A-P displacement ranges was observed as stability decreased (bipedal-firm vs bidepal-foam: $F(1.17)=9.56, p=0.004$; bipedal-firm vs unipedal-foam: $F(1.17)=9.56 ; p=0.007$ ) (Figures $4 D$ ). There were no significant changes in the M-L displacement ranges for the postural condition $(F(2,34)=0.59, p=0.50)$ or for the change times $(F(1.17)$ $=1.99, p=0.17$ ) (Figure $4 \mathrm{E}$ ). In the mean velocity of the upper trunk, a significant decrease was observed with late target changes compared to no change in all postural conditions $(F(1.17)=30.46, p \leq 0.001)$ (Figure 4F).

For the CoP control strategy, a modification was observed due to the postural condition and not due to the change times (Figures $4 \mathrm{E}-\mathrm{G}$ ). All parameters decreased as postural stability decreased. In A-P displacement, it was clearly observed that the most unstable positions decreased this range of displacement (bipedal-firm vs bipedal-foam: $F=109.3, p \leq 0.001$; bipedal-firm vs unipedal-foam: $F(1.17)$ $=109.3, p \leq 0.001$; bipedal-foam vs unipedal-foam: $\mathrm{F}(1.17)=109.3, \mathrm{p} \leq 0.001)$. The M-L displacement decreased for the unipedal-foam condition with respect to the other postures (bipedal-firm vs unipedalfoam: $F(1.17)=8.15, p=<0.001$; bipedal-foam vs unipedal-foam: $F(1.17)=8.15, p \leq 0.001)$. The mean velocity of the CoP decreased for the most unstable postural conditions with respect to the bipedal-firm (bipedal-firm vs bipedal-foam: $F(1.17)=30.69, p \leq 0.001$; bipedal-firm vs unipedal-foam: $F(1.17)=$ $30.69, p \leq 0.001$; bipedal-foam vs unipedal-foam: $F(1.17)=30.69, p \leq 0.001)$.

\section{Discussion}

We studied the effects of postural stability and target change on a pointing task under a double-step task paradigm. We found that postural instability did not affect pointing accuracy, but late changes in target position did. On the other hand, we found a strategy to decrease the oscillation and speed of the CoP during the pointing in more unstable conditions. The speed of the upper thorax and the finger decreased when there was a late change in the target; the A-P oscillation of the upper thorax decreased with greater instability; and the M-L displacement of the finger increased with a longer change time and instability. 
Regarding their performance in the pointing task, participants tended to respond more slowly when the visual target changed late, which is consistent with Ratcliff et al.'s findings ${ }^{25}$. They showed that subjects tended to increase the duration of the reach as a strategy to minimize error. Similar results have been shown by O'Reilly et al. ${ }^{15}$, where participants increased the time used when the target changes late $(600 \mathrm{~ms})$ but maintained similar levels of precision in the different target change times. Possibly, an increase in finger movement times would have given participants more feedback time to maintain a higher level of precision. In our results, we also found that precision in young subjects tended to be compromised when they had a shorter feedback time. This coincides with the literature that associates the last part of the reaching time with the effect of feedback on movement precision ${ }^{26}$.

Previous work has shown that quick responses are less accurate than slow responses ${ }^{27}$. Therefore, a better understanding of the relationship between task time and precision is needed for a clear picture of the reaching performance. We observed that a late target change compromised performance, affecting both precision and movement time.

Regarding the motion times, it was observed that when the target changed at 200 and $400 \mathrm{~ms}$, the subjects responded faster than when the condition was unchanged. This may have been due to the instruction given to participants, which allowed a comfortable speed, in contrast to other studies ${ }^{18}$ prioritizing the speed of response.

As for the effects of postural condition, the results obtained in the conditions of greater instability (bipedal-foam and unipedal-foam) did not alter the precision in relation to the most stable test (bipedalfirm). These results agree with what has been observed in previous studies ${ }^{4}$, which have shown that the movement times of the hand reaching moving stimulus do not vary under conditions of greater instability. This shows that the Central nervous system (CNS) is capable of taking into account the biomechanical limitations of an unstable posture when performing a voluntary movement. Previous works have concluded that the CNS can anticipate impending disturbances and correctly program postural adjustments in order to maintain balance and efficiently perform voluntary movement ${ }^{16}$.

It is known that in order to maintain posture while performing a reaching task, the CNS is constantly being informed of the environment by sensory inputs (visual, proprioceptive and vestibular) ${ }^{28}$. Additionally, the CNS is able to weigh which of the sensory inputs will be most used depending on the context of the task ${ }^{29}$. In the unipedal-foam condition, the proprioceptive system was being interfered with therefore, the vestibular system together with the visual system were essential to maintain a stable posture. Although the role of the vestibular system in balance is well known, recent studies have shown the role it plays in tasks of upper limbs, specifically in the planning and execution of reaching ${ }^{30,31}$. Under this context of online correction, vestibular cues function as a "spatial updater" and as a "contextual indicator" to select the appropriate motor commands for reaching during body movement, thus actively contributing with reaching correction ${ }^{31,32}$. Since vestibular signals have already been shown to be essential in the control of reaching movements with online correction, they could have been one of 
physiological mechanisms due to which the situations of postural instability did not alter the efficiency of the task in comparison with the more stable postures.

If we analyze the motion strategy used, we observed that both the control of the index finger and upper thorax were prioritized with respect to the CoP when postural instability increased. It was also observed that the control of the finger and the thorax decreased the velocity due to greater instability, while the finger increased its mediolateral trajectory due to a longer correction time and greater instability. We could suppose a complex global strategy exists where different effectors contribute with different postural and movement components to maintain pointing precision. This shows differentiated strategies according to the context with a prioritization of end-effector control ${ }^{33}$. Despite the recorded error, we cannot rule out that the subjects could perceive most of the trials as correct, even at change times of $600 \mathrm{~ms}$, which did not necessarily force them to optimize in relation to precision.

The postural condition affected how the motor strategy of the subjects was configured during the reaching task. As previously observed, humans tend to change their oscillatory patterns when they are subjected to situations that could generate threats to their stability ${ }^{34}$. As can be concluded from the results, the amount of CoP movement during the pointing movement decreases when the base of support becomes smaller, both in the ML and AP axes, a phenomenon that has already been reported in previous studies, where it was observed that a large percentage of the variability of the CoP and CoM movement depends on the base of support ${ }^{35}$. When the base of support becomes smaller, and therefore, more unstable, anticipatory postural adjustments are produced before the movement of the hand, whose final effect is the reduction of the oscillation and displacement of both the CoP and CoM, and on the contrary, postural adjustments increase when the base of support gets bigger; finally, we conclude that the initial postural configuration has a great influence on the APA patterns ${ }^{36}$. One hypothesis that explains this behavior is that when the postural task is more complicated, a restriction strategy that decreases postural sway occurs, reducing the necessary control to maintain a stable posture and allowing a more efficient hand motion ${ }^{37}$.

On the other hand, an important effect of the reaching task on postural control was observed. Indeed, during the pre-movement period of time, the unipedal-foam condition caused significant postural perturbation, resulting in a greater CoP displacement in the M-L plane. (fig. 2B). However, during the pointing execution period, the inverse phenomenon was observed, that is, the CoP displacement in the M$L$ plane decreased significantly and in proportion to the instability of the postural condition. This indicates that during each trial the subject alternated between two different postural strategies: one for the pre-movement period and one for reaching, depending on the postural condition of the task.

Contrary to expectations, the additional difficulty imposed by the online correction task did not seem to influence the postural challenges, even in the conditions of greatest attention load (change at $600 \mathrm{~ms}$, fig. $4 \mathrm{H})$. It has previously been observed that in reaching tasks with external forces on the upper limb these forces cause postural adaptations whose magnitude is directly proportional to the error induced by the disturbances ${ }^{38,39}$. 
The different postural conditions modified the M-L finger displacement and the A-P upper trunk displacement during the reaching movement. Movement velocity of the upper trunk and the finger was affected with later target changes $(600 \mathrm{~ms})$. In the study of Martin, et al. an increase in the anterior inclination of the trunk was seen, which could explain the decrease in A-P of the thorax ${ }^{40}$.

Contrary to what we expected, no significant differences were observed in the precision of the hand in the conditions of greater instability (unipedal-foam) and less correction time. Apparently the postural adjustments made by the system in the unipedal-foam conditions were sufficient to generate precise pointing. Previous work has shown that human beings, when subjected to instability situations, adopt postural strategies in order to avoid imminent falls $22,40,41$. We argue that in these situations of unipedal condition the young subjects could be adopting these strategies and optimizing the stability for the voluntary task. Future studies should evaluate how the muscular responses are generated under these experimental conditions or evaluate elderly people to determine the nature of instabilities in this population.

It is important to consider the specific paradigm that was used in this study, where we could observe that regardless of the environment in which the person is, they will be able to do the requested task with the greatest possible precision. In the perspective of the triad individual-task-environment ${ }^{42}$, the choice of the strategy that will allow solving the task is key. In a context of greater instability, a postural strategy will be used that allows reaching the target in the same way, but this strategy will be different in a context of greater postural stability, highlighting the role of the environment.

The CNS must constantly deal with uncertain scenarios. It is suggested that it is essential to use this uncertainty as a default parameter in natural situations that allows the correction posture control in different movements, including predictive elements ${ }^{40}$.

Despite the available evidence, the discussion is still open on whether the nature of the mechanisms for reaching with correction is anticipatory or corrective. It has been observed that in the bipedal position the trunk musculature is the first to be activated in the face of a reaching task with correction (33ms before the movement) ${ }^{22,40}$. Additionally, an early muscle activation of the deltoid and tibialis anterior (latencies less than $100 \mathrm{~ms}$ ) during a reaching with correction has been observed. These results suggest that in these online correction situations, postural adjustments are predictive in nature. They are not based on feedback from the moving limb, and they do not require cortical regulation ${ }^{21}$.

Other studies are in line with the optimal feedback control model proposed by Todorov and Jordan (2002) for reaching with correction ${ }^{43}$, in which feedback gains are adjusted based on estimation of environmental and limb status. Errors are corrected only if they interfere with the achievement of the task objective, but are otherwise ignored, following a "minimal intervention" principle. Another interesting feature of the optimal feedback control is that no desired hand trajectory is specified before the start of the movement, as it emerges flexibly based on continuous adjustments of the motor commands ${ }^{43,44}$. 
We propose a mixed model of feedforward with feedback integrated in a Bayesian control, depending on the time available for correction. When the time is sufficient (200 or $400 \mathrm{~ms}$ ), the anticipatory control would be complemented with the corrective one. This ensures the performance of the task and would not happen with lower correction times $(600 \mathrm{~ms})$. Complementary models have previously been proposed between anticipation and correction under a Bayesian control vision where the anticipatory model is combined with the corrective one as a sensory verification according to the ongoing task results $11,45,46$.

In conclusion, the CNS has the ability to use different strategies that allow the achievement of a pointing task despite disturbances in postural stability, which is achieved by changing the posture stabilization strategy. On the other hand, correcting the pointing trajectory is more difficult when the target changes occur later, using changes in the strategy in the endpoint control to be able to correct the reaching trajectory. There is probably a weighting of anticipatory and corrective models according to the postural and temporal context of the changes that occurred during the task. Thus, we argue that the postural condition does not affect the adequate availability of these models; however, the late change time decreases the possibility of using feedback, affecting the time-precision relationship in the task.

\section{Methodology}

\section{Participants}

19 participants (10 men and 9 women) were invited to this study. The subjects had an average age of 25 \pm 2.1 years without health alterations that could alter their performance in the task (corrected or normal vision, without neurological deterioration, pain, or biomechanical alterations). All experimental protocols were in accordance with the Declaration of Helsinki and approved by the "Central Metropolitan Health Service of Chile" ethics committee. All participants signed the informed consent approved by the ethics committee.

\section{Task}

A pointing task was designed toward a target displayed in the center of a touch screen (Figure 1). The target ( $0.8 \mathrm{~cm}$ diameter circle) changed from black to red when the subject released a button located on the thorax (xiphoid process). Then it could be randomly held in the center or moved to 4 positions (up, down, right or left) $4.5 \mathrm{~cm}$ from the center position. For the tests with the change of target position, this occurred with 3 latencies $(200,400$ or 600 ms) with respect to the onset of pointing. The task was performed in 3 postural conditions: bipedal on a firm surface (bipedal-firm), bipedal on foam (bipedalfoam), and unipedal on foam (unipedal-foam). The high-density foam AIREX® Balance-pad Elite was used with dimensions of $19.7^{\prime \prime} \times 16.1^{\prime \prime} \times 2.4$ " and a thickness of $6 \mathrm{~cm}$.

\section{Data collection}

The presentation of the stimuli was programmed in Python 2.7 (Copyright (C) 1990-2022, Python Software Foundation) using the Pygame library. The screen was a 10-inch (21.6 x $13.5 \mathrm{~cm}$, waveshare brand) LED 
touchscreen controlled by a Raspberry pi 3B microcontroller.

By means of electrical pulses, the moment in which the participants released the chest button was recorded. By recording the activity of the touch screen, the moment of contact and the place of contact were recorded on the screen, in $x, y$ coordinates in pixels $(800 \times 600)$ at a sampling rate of $100 \mathrm{~Hz}$.

Using 10 infrared cameras VICON brand (Vantage V5, Oxford Metrics Group, Oxford, UK) and reflective markers placed in different body segments of the upper body, we recorded the kinematics of the pointing at a sampling rate of $200 \mathrm{~Hz}$. The used model was the biomechanical "Upper limbs" of the Nexus program. Additionally, a marker external to this model was used to represent the tip of the index finger and thus have the registration of the finger and upper thorax (spinous process $\mathrm{C} 7$ ).

The center of pressure was recorded during the task using a triaxial force platform (model FP4060; Bertec, Columbus, $\mathrm{OH}$ ) at a sampling rate of $1000 \mathrm{~Hz}$.

\section{Protocol}

Before carrying out the experimental task, each participant conducted a session of 15 trials toward the central target, without changes or displacements, in a stable bipedal posture. The objective was to have baseline values to perform the normalization of the data according to each participant.

For each task, participants were positioned with the index finger of their dominant hand by pressing a starting button located on the thorax(xiphoid process). The subjects were asked to achieve the static target as accurately and naturally as possible. Then they were subjected to three conditions, which ranged from least to greatest postural instability: bipedal-firm, bipedal-foam, and unipedal-foam. The participants were barefoot on the force platform. In the bipedal condition, the feet were adjusted for each participant according to the projection of the distances between the anterior superior iliac spines. This standard position for each subject was marked through a tape on the platform, ensuring a stable position for each trial in each postural condition. On the other hand, in the unipedal posture, the subjects were asked to bring one of their legs back in a $90^{\circ}$ knee flexion while keeping the lower limb that they considered dominant on the surface.

The center of the screen was aligned with the center between both eyes. The distance from the screen was $130 \%$ of the acromial-fingertip distance, considering the total reach distance with the dominant arm extended (reaching distance). This distance was chosen to generate postural responses to a reaching task in the bipedal posture, without generating a step response ${ }^{4}$ (Figure 1).

Each participant performed 150 trials for each postural condition, including static and corrected tasks. The stimuli were presented randomly, maintaining a proportion of $20 \%$ without a change in the target and $80 \%$ with the change in the target. In that $80 \%$, there was a homogeneous distribution for each latency and direction. Between each postural condition, the subject was allowed to rest to avoid fatigue. Additionally, in the unipedal-foam test (the most demanding condition), after every 10 trials, the subject 
was allowed to lower the leg and rest so that the fatigue of the test would not affect performance. Because of fatigue, we always kept the order of postural conditions from least to greatest complexity.

\section{Variables}

The processing of variables was performed in their own codes in the Python 3.6 language. Movement time was estimated between the release time of the button on the xiphoid process until the touch of the screen.

The execution error was calculated as the difference between the position of the center of the target and the place touched in pixels. The pixels were then transformed into centimeters based on the dimensions of the screen.

Both the time and the error were normalized to the $z$-score with respect to the average performance of the 15 baseline trials.

To assess the overall performance based on Fitts' Law (Fitts, $1954^{27}$ ), we computed a composite variable of time and error as an average of the previously described Z scores. Higher values of this variable represent a worse performance, given by a longer execution time and a greater error on the screen.

The positions and trajectories of the finger and the upper trunk were estimated directly from the registration of the markers in the three dimensions (Figure 1B). The position and trajectory of the center of pressure (CoP) were estimated from the filtered signal of the force platform in two dimensions using a Butterworth low pass filter of $4 \mathrm{~Hz}$ and of fourth-order (Figure 1C).

All signals were temporally segmented into two time windows. First, the Pre-Movement window corresponds to $500 \mathrm{~ms}$ prior to the release of the thorax button, and then the Movement window is defined between the release of the thorax button and the touching of the screen. In these windows, the range of displacement of the finger, trunk, and CoP was estimated as the difference between the 5th and 95th percentiles of the instantaneous position in the anteroposterior (A-P) and mediolateral (M-L) planes. Higher ranges imply a greater displacement in each of the planes. In addition, the average speed of these signals was calculated, performing the first derivative of the position $\left(\mathrm{mm} / \mathrm{s}^{2}\right)$ and then calculating the average of these time series.

\section{Statistical analysis}

Each variable was described by the mean and standard deviation of the sample. To this end, the performance of each participant was averaged for the trials and grouped according to postural condition and target correction time. For inferential statistics, the repeated-measures analysis of variance (ANOVA) was used with one (experimental condition) or two factors (correction time), as appropriate. Both normality and sphericity assumptions were tested. According to the result of the Mauchly test, the Greenhouse - Geisser correction was applied. Post-hoc analysis of multiple comparisons considered Holm's correction. For all purposes, the p-value was set at 0.05 . All analyses were performed in R and 
Rstudio (RStudio Team (2020). RStudio: Integrated Development Environment for R. RStudio, PBC, Boston, MA).

\section{Declarations}

\section{Data availability}

Data will be available in Physionet (Burgos, P. (2022). Effects of balance and timing constraints on performance during a pointing task with target uncertainty ). Only logged-in users who sign the specified data use agreement can access the files. The License (for files) is: PhysioNet Restricted Health Data License 1.5.0

\section{Acknowledgments}

We want to thank the High Performance Center of Chile for contributing with the facilities and devices needed to carry out this investigation.

\section{Funding}

PIB was supported by a Fondecyt Grant \#11181337 from the National Agency for Research and Development(ANID), Government of Chile.

\section{Author contributions}

CMJ contributed to the conception, design and interpretation of the data, and the drafting. CMJ revised, approved, and is accountable for all aspects of the work.

JJM contributed to the analysis, interpretation of data, critical revision. JJM approved of the work and is accountable for all aspects of the work.

TBM contributed to the drafting, revising, analysis and interpretation of the data. TBM approved of the work and is accountable for all aspects of the work.

FAL contributed to the drafting, revising, analysis and interpretation of the data. FAL approved of the work and is accountable for all aspects of the work.

JCP contributed to the statistical analysis, interpretation of data, critical revision. JCP approved of the work and is accountable for all aspects of the work.

CMP contributed to the acquisition, interpretation of data, critical revision. CMP approved of the work and is accountable for all aspects of the work.

JT contributed to the statistical analysis, interpretation of data, critical revision. JT approved and is accountable for all aspects of the work. 
EAM contributed with data collection and revision. EAM approved and is accountable for all aspects of the work.

PIB contributed to the conception, design analysis, interpretation of the data, and drafting. PIB revised, approved, and is accountable for all aspects of the work.

\section{Competing interests}

The author(s) declare no competing interests.

\section{References}

1. Gomi, H. Implicit online corrections of reaching movements. Curr. Opin. Neurobiol. 18, 558-564 (2008).

2. van Beers, R. J., Baraduc, P. \& Wolpert, D. M. Role of uncertainty in sensorimotor control. Philos. Trans. R. Soc. B Biol. Sci. 357, 1137-1145 (2002).

3. Jacobs, J. V. \& Horak, F. B. Cortical control of postural responses. J. Neural Transm. Vienna Austria 1996 114, 1339-1348 (2007).

4. Hua, S., Leonard, J. A., Hilderley, A. J. \& Stapley, P. J. Postural configuration does not alter unperturbed or perturbed reach movement kinematics. Exp. Brain Res. 227, 63-78 (2013).

5. Jovancevic-Misic, J. \& Hayhoe, M. Adaptive gaze control in natural environments. J. Neurosci. Off. J. Soc. Neurosci. 29, 6234-6238 (2009).

6. Won, J. \& Hogan, N. Stability properties of human reaching movements. Exp. Brain Res. 107, 125136 (1995).

7. Saunders, J. A. \& Knill, D. C. Humans use continuous visual feedback from the hand to control fast reaching movements. Exp. Brain Res. 152, 341-352 (2003).

8. Yeomans, M., Yan, S., Hondzinski, J. M. \& Dalecki, M. Eye-hand decoupling decreases visually guided reaching independently of posture but reduces sway while standing: Evidence for supra-postural control. Neurosci. Lett. 752, 135833 (2021).

9. Bizzi, E., Mussa-Ivaldi, F. A. \& Giszter, S. Computations Underlying the Execution of Movement: A Biological Perspective. Science 253, 287-291 (1991).

10. Wolpert, D. M. \& Ghahramani, Z. Computational principles of movement neuroscience. Nat. Neurosci. 3 Suppl, 1212-7 (2000).

11. Wolpert, D. M. Computations in Sensorimotor Learning. Cold Spring Harb. Symp. Quant. Biol. 79, 9398 (2014).

12. Sabes, P. N. The planning and control of reaching movements. Curr. Opin. Neurobiol. 10, 740-746 (2000).

13. Bringoux, L. et al. Double-Step Paradigm in Microgravity: Preservation of Sensorimotor Flexibility in Altered Gravitational Force Field. Front. Physiol. 11, 377 (2020). 
14. Desmurget, null \& Grafton, null. Forward modeling allows feedback control for fast reaching movements. Trends Cogn. Sci. 4, 423-431 (2000).

15. O'Rielly, J. L. \& Ma-Wyatt, A. Changes to online control and eye-hand coordination with healthy ageing. Hum. Mov. Sci. 59, 244-257 (2018).

16. Zhang, Y., Brenner, E., Duysens, J., Verschueren, S. \& Smeets, J. B. J. Postural responses to target jumps and background motion in a fast pointing task. Exp. Brain Res. 236, 1573-1581 (2018).

17. Paulignan, Y., MacKenzie, C., Marteniuk, R. \& Jeannerod, M. Selective perturbation of visual input during prehension movements. 1. The effects of changing object position. Exp. Brain Res. 83, 502512 (1991).

18. Sarlegna, F. R. \& Mutha, P. K. The influence of visual target information on the online control of movements. Vision Res. 110, 144-154 (2015).

19. Day, B. L. \& Lyon, I. N. Voluntary modification of automatic arm movements evoked by motion of a visual target. Exp. Brain Res. 130, 159-168 (2000).

20. Soechting, J. F. \& Lacquaniti, F. Modification of trajectory of a pointing movement in response to a change in target location. J. Neurophysiol. 49, 548-564 (1983).

21. Leonard, J. A., Gritsenko, V., Ouckama, R. \& Stapley, P. J. Postural adjustments for online corrections of arm movements in standing humans. J. Neurophysiol. 105, 2375-2388 (2011).

22. Fautrelle, L., Prablanc, C., Berret, B., Ballay, Y. \& Bonnetblanc, F. Pointing to double-step visual stimuli from a standing position: very short latency (express) corrections are observed in upper and lower limbs and may not require cortical involvement. Neuroscience 169, 697-705 (2010).

23. Laessoe, U., Hoeck, H. C., Simonsen, O. \& Voigt, M. Residual attentional capacity amongst young and elderly during dual and triple task walking. Hum. Mov. Sci. 27, 496-512 (2008).

24. Patel, P. J. \& Bhatt, T. Attentional demands of perturbation evoked compensatory stepping responses: examining cognitive-motor interference to large magnitude forward perturbations. J. Mot. Behav. 47, 201-210 (2015).

25. Ratcliff, R., Thapar, A. \& McKoon, G. Aging and individual differences in rapid two-choice decisions. Psychon. Bull. Rev. 13, 626-635 (2006).

26. Kimura, D., Kadota, K. \& Kinoshita, H. The impact of aging on the spatial accuracy of quick corrective arm movements in response to sudden target displacement during reaching. Front. Aging Neurosci. 7, 182 (2015).

27. Fitts, P. M. The information capacity of the human motor system in controlling the amplitude of movement. 1954. J. Exp. Psychol. Gen. 121, 262-269 (1992).

28. Peterka, R. J., Wall, C. \& Kentala, E. Determining the effectiveness of a vibrotactile balance prosthesis. J. Vestib. Res. Equilib. Orientat. 16, 45-56 (2006).

29. Peterka, R. J. \& Loughlin, P. J. Dynamic regulation of sensorimotor integration in human postural control. J. Neurophysiol. 91, 410-423 (2004). 
30. Peterka, R. J. Sensorimotor integration in human postural control. J. Neurophysiol. 88, 1097-1118 (2002).

31. DiZio, P. \& Lackner, J. R. Coriolis-Force-Induced Trajectory and Endpoint Deviations in the Reaching Movements of Labyrinthine-Defective Subjects. J. Neurophysiol. 85, 784-789 (2001).

32. Bresciani, J.-P. et al. On-line versus off-line vestibular-evoked control of goal-directed arm movements. Neuroreport 13, 1563-6 (2002).

33. Horak, F. B., Nutt, J. G. \& Nashner, L. M. Postural inflexibility in parkinsonian subjects. J. Neurol. Sci. 111, 46-58 (1992).

34. Zaback, M., Luu, M. J., Adkin, A. L. \& Carpenter, M. G. Selective preservation of changes to standing balance control despite psychological and autonomic habituation to a postural threat. Sci. Rep. 11, 384 (2021).

35. Watanabe, T., Saito, K., Ishida, K., Tanabe, S. \& Nojima, I. Coordination of plantar flexor muscles during bipedal and unipedal stances in young and elderly adults. Exp. Brain Res. 236, 1229-1239 (2018).

36. Kaminski, T. R. \& Simpkins, S. The effects of stance configuration and target distance on reaching. I. Movement preparation. Exp. Brain Res. 136, 439-446 (2001).

37. Ilha, J., Abou, L., Romanini, F., Dall Pai, A. C. \& Mochizuki, L. Postural control and the influence of the extent of thigh support on dynamic sitting balance among individuals with thoracic spinal cord injury. Clin. Biomech. Bristol Avon 73, 108-114 (2020).

38. Pienciak-Siewert, A., Horan, D. P. \& Ahmed, A. A. Trial-to-trial adaptation in control of arm reaching and standing posture. J. Neurophysiol. 116, 2936-2949 (2016).

39. Horak, F. B. \& Diener, H. C. Cerebellar control of postural scaling and central set in stance. J. Neurophysiol. 72, 479-493 (1994).

40. Martin, O., Teasdale, N., Simoneau, M., Corbeil, P. \& Bourdin, C. Pointing to a target from an upright position in human: Tuning of postural responses when there is target uncertainty. Neurosci. Lett. 281, 53-6 (2000).

41. Tassani, S., Font-Llagunes, J. M., González Ballester, M. Á. \& Noailly, J. Muscular tension significantly affects stability in standing posture. Gait Posture 68, 220-226 (2019).

42. Shumway-Cook, A. \& Woollacott, M. H. Motor Control: Translating Research into Clinical Practice. (2011).

43. Todorov, E. \& Jordan, M. I. Optimal feedback control as a theory of motor coordination. Nat. Neurosci. 5, 1226-1235 (2002).

44. Diedrichsen, J., Shadmehr, R. \& Ivry, R. B. The coordination of movement: optimal feedback control and beyond. Trends Cogn. Sci. 14, 31-39 (2010).

45. Wolpert, D. M. Probabilistic models in human sensorimotor control. Hum. Mov. Sci. 26, 511-24 (2007). 
46. Körding, K. P. \& Wolpert, D. M. Bayesian integration in sensorimotor learning. Nature 427, 244-7 (2004).

\section{Tables}

Table 1. Multiple comparisons of pointing performance on the screen in the different posture conditions and change times.

\begin{tabular}{|c|c|c|c|c|}
\hline \multirow[t]{2}{*}{ Posture condition } & \multirow[t]{2}{*}{ Change Time (ms) } & \multirow{2}{*}{$\begin{array}{l}\text { Motion time } \\
\text { (z score) } \\
\text { p-value }\end{array}$} & \multirow{2}{*}{$\begin{array}{l}\text { Error } \\
\text { (z score) } \\
\text { p-value }\end{array}$} & Error \& motion time (z score) \\
\hline & & & & p-value \\
\hline \multirow[t]{6}{*}{ Bipedal-firm } & 0 vs 200 & 0.014 & 0.051 & 0.82 \\
\hline & 0 vs 400 & 0.15 & 0.03 & 0.36 \\
\hline & 0 vs 600 & 0.0011 & 0.023 & 0.004 \\
\hline & 200 vs 400 & 0.031 & 0.87 & 0.33 \\
\hline & 200 vs 600 & $<0.001$ & 0.03 & 0.004 \\
\hline & 400 vs 600 & $<0.001$ & 0.03 & 0.005 \\
\hline \multirow[t]{6}{*}{ Bipedal-foam } & 0 vs 200 & 0.055 & 1 & 0.63 \\
\hline & 0 vs 400 & 0.87 & 1 & 0.63 \\
\hline & 0 vs 600 & 0.039 & 0.05 & 0.019 \\
\hline & 200 vs 400 & 0.012 & 1 & 0.23 \\
\hline & 200 vs 600 & 0.004 & 0.02 & 0.004 \\
\hline & 400 vs 600 & 0.028 & 0.04 & 0.015 \\
\hline \multirow[t]{6}{*}{ Unipedal-foam } & 0 vs 200 & 0.014 & 0.34 & 0.39 \\
\hline & 0 vs 400 & 0.043 & 0.15 & 0.39 \\
\hline & 0 vs 600 & 0.005 & 0.01 & 0.002 \\
\hline & 200 vs 400 & 0.039 & 0.34 & 0.025 \\
\hline & 200 vs 600 & 0.001 & 0.058 & 0.003 \\
\hline & 400 vs 600 & $<0.001$ & 0.15 & 0.012 \\
\hline
\end{tabular}

\section{Figures}



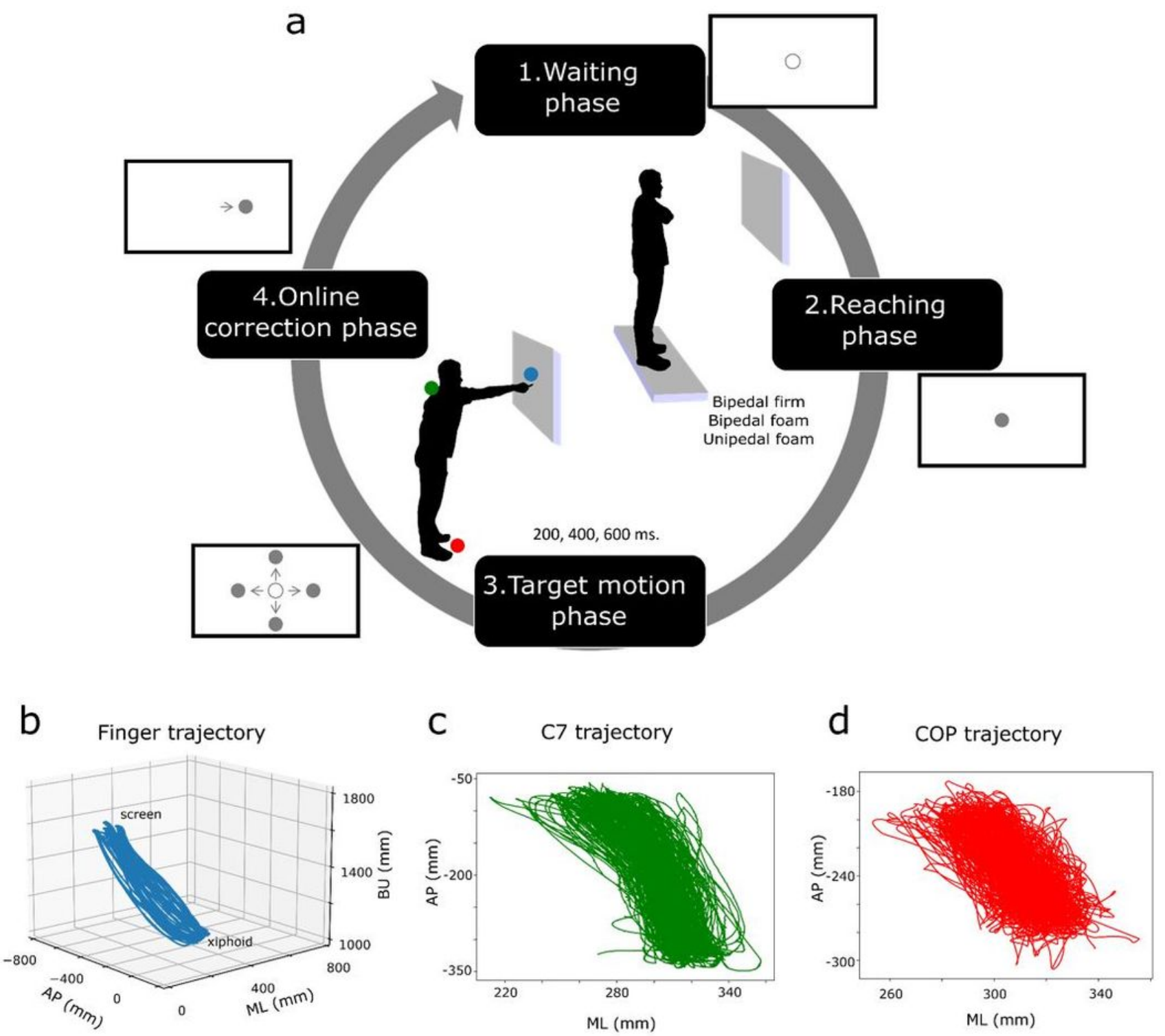

Figure 1

Experimental design and source data. a. Subjects were asked to perform a pointing task toward a static target located in the center of the screen. After the onset of the movement, the initial target was changed to a new location (up, down, right, or left) in three different change times $(200,400$ or $600 \mathrm{~ms})$. The task was repeated 150 trials in three different postural conditions (bipedal-firm, bipedal-foam, and unipedalfoam), b. The three-dimensional trajectory of the index finger marker of a participant performing the pointing tests in the bipedal-firm condition from the xiphoid process to the screen (150 trials). c. The twodimensional trajectory of the upper thorax marker (C7 process) of the same subject and conditions of figure $b$. d. The two-dimensional trajectory of the center of pressure (CoP) of the same subject and conditions of figure $b$. The colored dots in figure $a$ are represented in the same colors as the figures $b$ - $d$. 

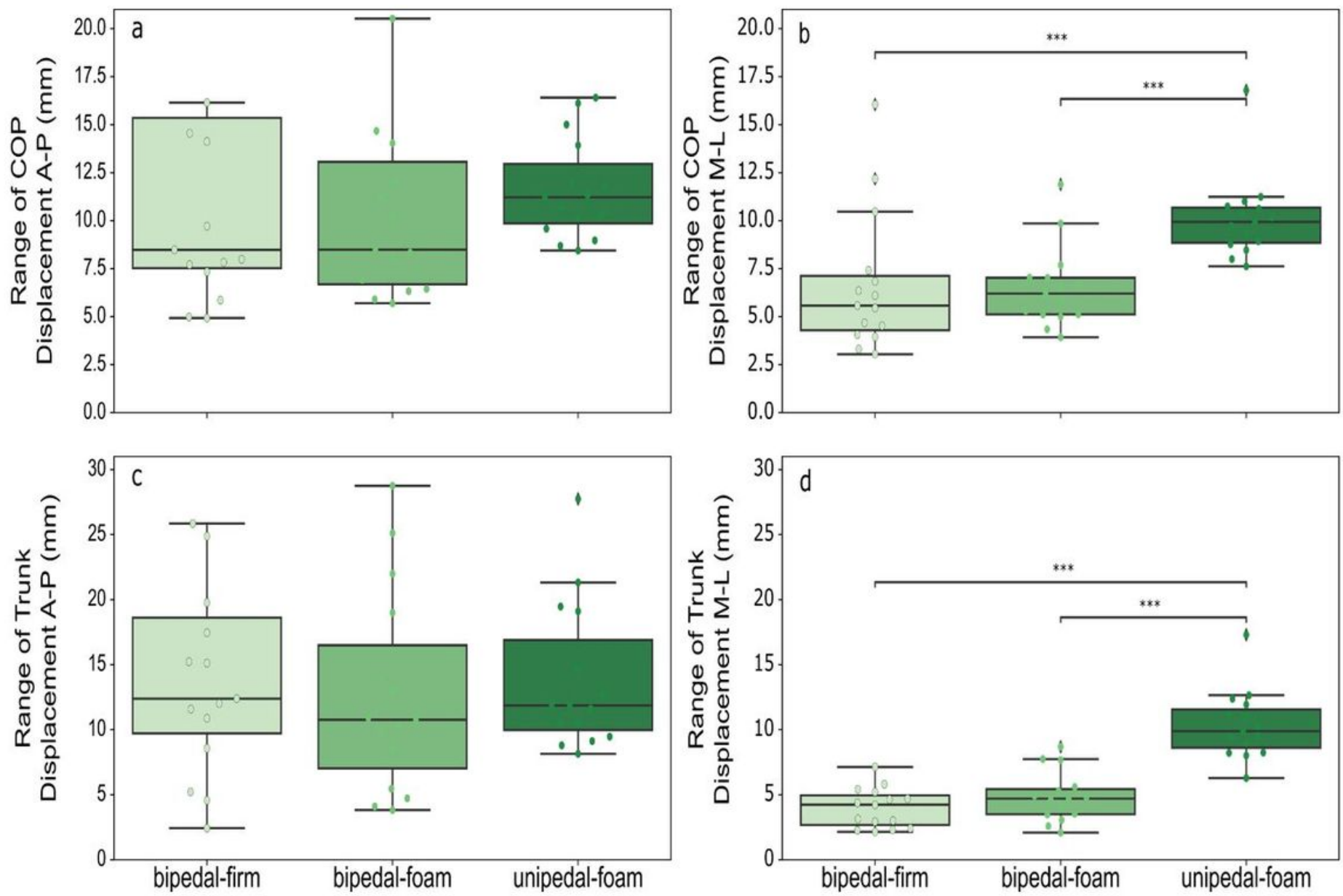

Figure 2

Postural stability in the pre-movement window $(-500 \mathrm{~ms}$ to $0 \mathrm{~ms})$. a. Range of displacement of the center of pressure (CoP) in the anteroposterior plane (A-P). b. Range of displacement of the center of pressure $(\mathrm{CoP})$ in the mediolateral plane (M-L). c. Range of displacement of the upper thorax (C7) in the A-P plane. d. Range of upper thorax displacement (C7) in the mediolateral M-L plane $(* p \leq 0.05, * \star p \leq 0.01, * \star *$ $p \leq 0.001, * * * * p \leq 0.0001)$. 

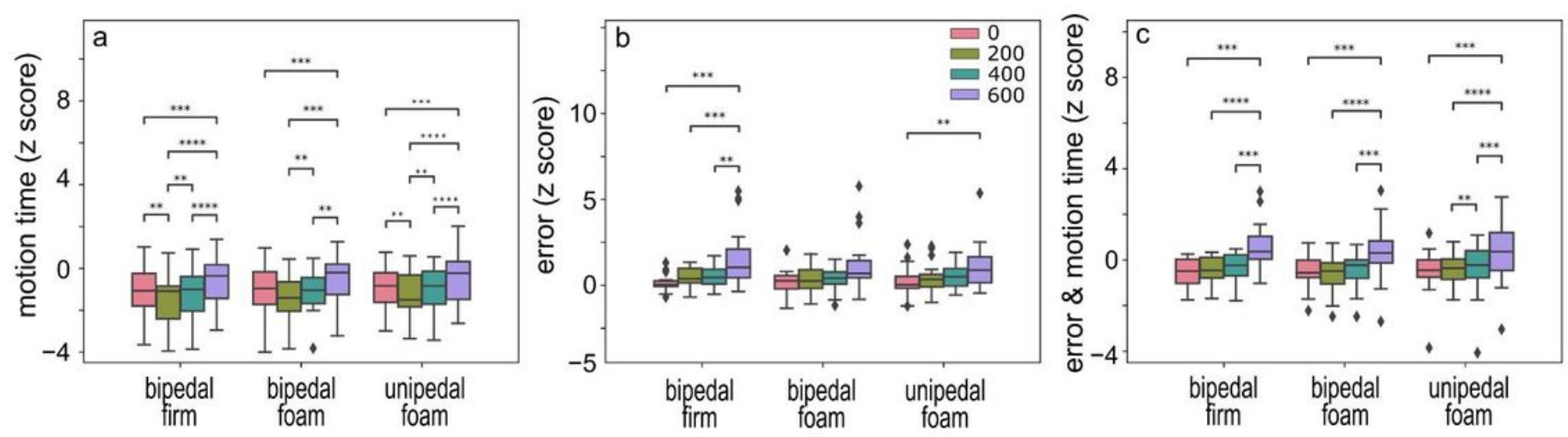

Figure 3

Pointing performance on the screen in the different posture conditions and change times. a. Motion time normalized with respect to baseline performance. $\mathbf{b}$. Spatial error of pointing on the screen normalized with respect to baseline performance. c. Composite metric of error and motion time (average of a and b), $(* p \leq 0.05, * \star p \leq 0.01, * \star * p \leq 0.001, * \star \star \star p \leq 0.0001)$. 

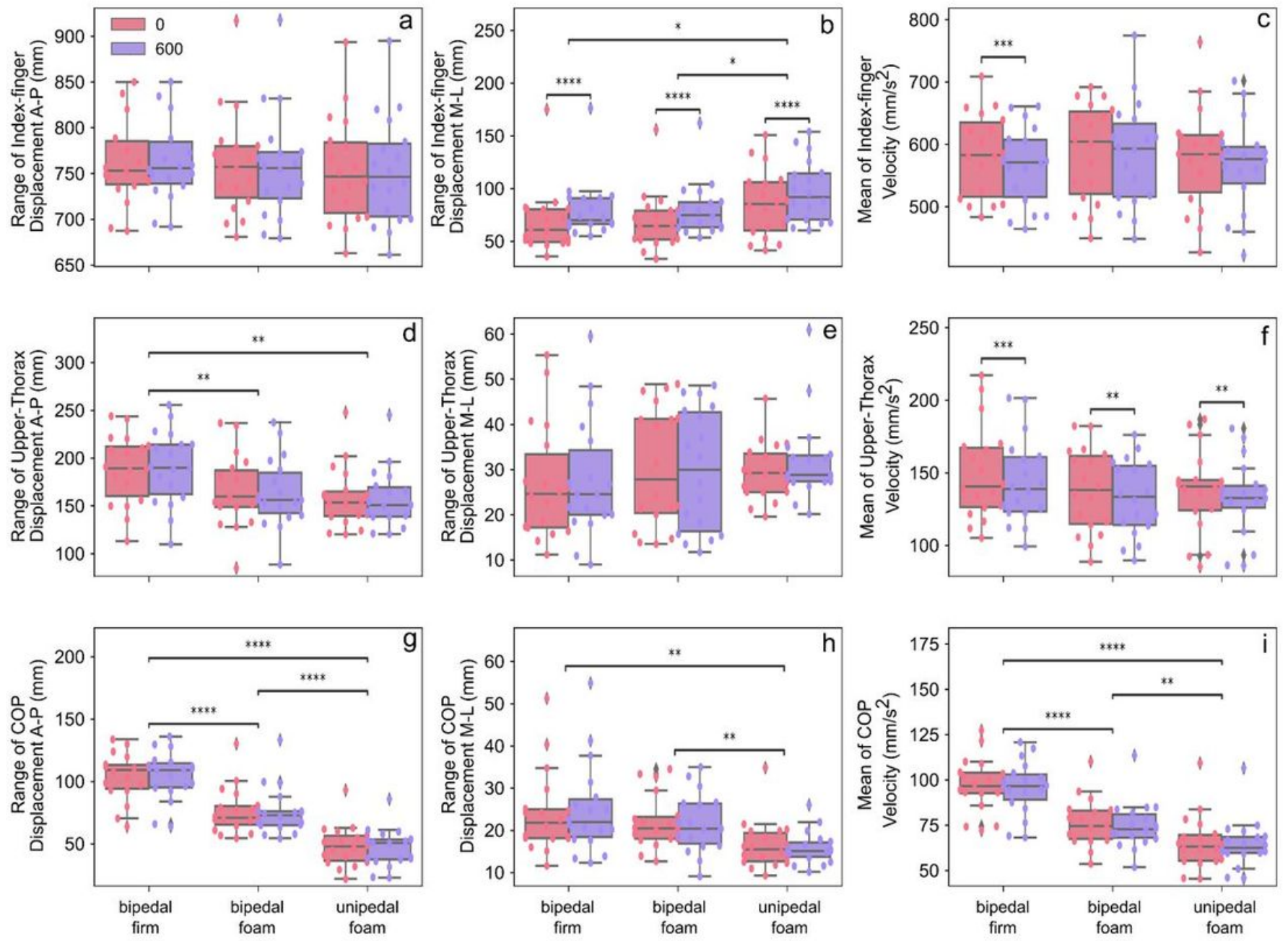

Figure 4

Motion and posture strategy during pointing. a-c. A-P \& M-L displacement ranges and index finger velocity. d-f. A-P \& M-L displacement ranges and upper thorax velocity (C7). g-i. A-P \& M-L displacement ranges and CoP velocity $(* p \leq 0.05, * \star p \leq 0.01, * \star * p \leq 0.001, * \star \star * p \leq 0.0001)$. 